Eze R. Nwaeze • Delfim F. M. Torres(i)

\title{
Chain rules and inequalities for the BHT fractional calculus on arbitrary timescales
}

Received: 25 February 2016 / Accepted: 28 November 2016 / Published online: 27 December 2016

(C) The Author(s) 2016. This article is published with open access at Springerlink.com

\begin{abstract}
We develop the Benkhettou-Hassani-Torres fractional (noninteger order) calculus on timescales by proving two chain rules for the $\alpha$-fractional derivative and five inequalities for the $\alpha$-fractional integral. The results coincide with well-known classical results when the operators are of (integer) order $\alpha=1$ and the timescale coincides with the set of real numbers.
\end{abstract}

Mathematics Subject Classification $26 \mathrm{~A} 33 \cdot 26 \mathrm{D} 10 \cdot 26 \mathrm{E} 70$

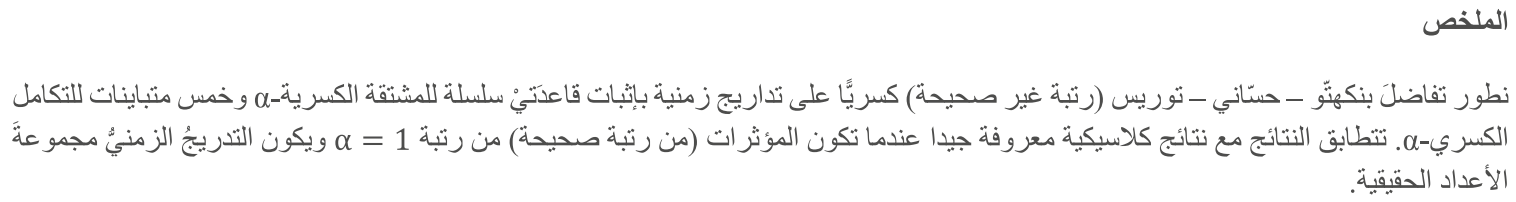

\section{Introduction}

The study of fractional (noninteger order) calculus on timescales is a subject of strong current interest [1-4]. Recently, Benkhettou, Hassani and Torres introduced a (local) fractional calculus on arbitrary timescales $\mathbb{T}$ (called here the BHT fractional calculus) based on the $T_{\alpha}$ differentiation operator and the $\alpha$-fractional integral [5]. The Hilger timescale calculus [6] is then obtained as a particular case, by choosing $\alpha=1$. In this paper, we develop the BHT timescale fractional calculus initiated in [5]. Precisely, we prove two different chain rules for the fractional derivative $T_{\alpha}$ (Theorems 3.1 and 3.3) and several inequalities for the $\alpha$-fractional integral: Hölder's inequality (Theorem 3.4), Cauchy-Schwarz's inequality (Theorem 3.5), Minkowski's inequality (Theorem 3.7), generalized Jensen's fractional inequality (Theorem 3.8) and a weighted fractional HermiteHadamard inequality on timescales (Theorem 3.9).

The paper is organized as follows. In Sect. 2, we recall the basics of the the BHT fractional calculus. Our results are then formulated and proved in Sect. 3.

E. R. Nwaeze

Department of Mathematics, Tuskegee University, Tuskegee, AL 36088, USA

E-mail: enwaeze@mytu.tuskegee.edu

D. F. M. Torres $(\varangle)$

CIDMA, Department of Mathematics, University of Aveiro, 3810-193 Aveiro, Portugal

E-mail: delfim@ua.p 


\section{Preliminaries}

We briefly recall the necessary notions from the BHT fractional calculus [5]: fractional differentiation and fractional integration on timescales. For an introduction to the timescale theory we refer the reader to the book [6].

Definition 2.1 (See [5]) Let $\mathbb{T}$ be a timescale, $f: \mathbb{T} \rightarrow \mathbb{R}, t \in \mathbb{T}^{\kappa}$, and $\alpha \in(0,1]$. For $t>0$, we define $T_{\alpha}(f)(t)$ to be the number (provided it exists) with the property that, given any $\epsilon>0$, there is a $\delta$-neighbourhood $\mathcal{V}_{t}=(t-\delta, t+\delta) \cap \mathbb{T}$ of $t, \delta>0$, such that $\left|[f(\sigma(t))-f(s)] t^{1-\alpha}-T_{\alpha}(f)(t)[\sigma(t)-s]\right| \leq \epsilon|\sigma(t)-s|$ for all $s \in \mathcal{V}_{t}$. We call $T_{\alpha}(f)(t)$ the $\alpha$-fractional derivative of $f$ of order $\alpha$ at $t$, and we define the $\alpha$-fractional derivative at 0 as $T_{\alpha}(f)(0):=\lim _{t \rightarrow 0^{+}} T_{\alpha}(f)(t)$.

If $\alpha=1$, then we obtain from Definition 2.1 the Hilger delta derivative of timescales [6]. The $\alpha$-fractional derivative of order zero is defined by the identity operator: $T_{0}(f):=f$. The basic properties of the $\alpha$-fractional derivative on timescales are given in [5], together with several illustrative examples. Here we just recall the item (iv) of Theorem 4 in [5], which is needed in the proof of our Theorem 3.1.

Theorem 2.2 (See [5]) Let $\alpha \in(0,1]$ and $\mathbb{T}$ be a timescale. Assume $f: \mathbb{T} \rightarrow \mathbb{R}$ and let $t \in \mathbb{T}^{\kappa}$. If $f$ is $\alpha$-fractional differentiable of order $\alpha$ at $t$, then

$$
f(\sigma(t))=f(t)+\mu(t) t^{\alpha-1} T_{\alpha}(f)(t) .
$$

The other main operator of [5] is the $\alpha$-fractional integral of $f: \mathbb{T} \rightarrow \mathbb{R}$, defined by

$$
\int f(t) \Delta^{\alpha} t:=\int f(t) t^{\alpha-1} \Delta t
$$

where the integral on the right-hand side is the usual Hilger delta-integral of timescales [5, Def. 26]. If $F_{\alpha}(t):=\int f(t) \Delta^{\alpha} t$, then one defines the Cauchy $\alpha$-fractional integral by $\int_{a}^{b} f(t) \Delta^{\alpha} t:=F_{\alpha}(b)-F_{\alpha}(a)$, where $a, b \in \mathbb{T}$ [5, Def. 28]. The interested reader can find the basic properties of the Cauchy $\alpha$-fractional integral in [5]. Here we are interested to prove some fractional integral inequalities on timescales. For that, we use some of the properties of [5, Theorem 31].

Theorem 2.3 (Cf. Theorem 31 of [5]) Let $\alpha \in(0,1], a, b, c \in \mathbb{T}, \gamma \in \mathbb{R}$, and $f, g$ be two rd-continuous functions. Then,

(i) $\int_{a}^{b}[f(t)+g(t)] \Delta^{\alpha} t=\int_{a}^{b} f(t) \Delta^{\alpha} t+\int_{a}^{b} g(t) \Delta^{\alpha} t$;

(ii) $\int_{a}^{b}(\gamma f)(t) \Delta^{\alpha} t=\gamma \int_{a}^{b} f(t) \Delta^{\alpha} t$;

(iii) $\int_{a}^{b} f(t) \Delta^{\alpha} t=-\int_{b}^{a} f(t) \Delta^{\alpha} t$;

(iv) $\int_{a}^{b} f(t) \Delta^{\alpha} t=\int_{a}^{c} f(t) \Delta^{\alpha} t+\int_{c}^{b} f(t) \Delta^{\alpha} t$;

(v) if there exist $g: \mathbb{T} \rightarrow \mathbb{R}$ with $|f(t)| \leq g(t)$ for all $t \in[a, b]$, then $\left|\int_{a}^{b} f(t) \Delta^{\alpha} t\right| \leq \int_{a}^{b} g(t) \Delta^{\alpha} t$.

\section{Main results}

The chain rule, as we know it from the classical differential calculus, does not hold for the BHT fractional calculus. A simple example of this fact has been given in [5, Example 20]. Moreover, it has been shown in [5, Theorem 21], using the mean value theorem, that if $g: \mathbb{T} \rightarrow \mathbb{R}$ is continuous and fractional differentiable of order $\alpha \in(0,1]$ at $t \in \mathbb{T}^{\kappa}$ and $f: \mathbb{R} \rightarrow \mathbb{R}$ is continuously differentiable, then there exists $c \in[t, \sigma(t)]$ such that $T_{\alpha}(f \circ g)(t)=f^{\prime}(g(c)) T_{\alpha}(g)(t)$. In Sect. 3.1, we provide two other chain rules. Then, in Sect. 3.2, we prove some fractional integral inequalities on timescales.

\subsection{Fractional chain rules on timescales}

Theorem 3.1 (Chain Rule I) Let $f: \mathbb{R} \rightarrow \mathbb{R}$ be continuously differentiable, $\mathbb{T}$ be a given timescale and $g: \mathbb{T} \rightarrow \mathbb{R}$ be $\alpha$-fractional differentiable. Then, $f \circ g: \mathbb{T} \rightarrow \mathbb{R}$ is also $\alpha$-fractional differentiable with 


$$
T_{\alpha}(f \circ g)(t)=\left[\int_{0}^{1} f^{\prime}\left(g(t)+h \mu(t) t^{\alpha-1} T_{\alpha}(g)(t)\right) d h\right] T_{\alpha}(g)(t) .
$$

Proof We begin by applying the ordinary substitution rule from calculus:

$$
\begin{aligned}
f(g(\sigma(t)))-f(g(s)) & =\int_{g(s)}^{g(\sigma(t))} f^{\prime}(\tau) \mathrm{d} \tau \\
& =[g(\sigma(t))-g(s)] \int_{0}^{1} f^{\prime}(h g(\sigma(t))+(1-h) g(s)) \mathrm{d} h .
\end{aligned}
$$

Let $t \in \mathbb{T}^{\kappa}$ and $\epsilon>0$. Since $g$ is $\alpha$-fractional differentiable at $t$, we know from Definition 2.1 that there exists a neighbourhood $U_{1}$ of $t$ such that

$$
\left|[g(\sigma(t))-g(s)] t^{1-\alpha}-T_{\alpha}(g)(t)(\sigma(t)-s)\right| \leq \epsilon^{*}|\sigma(t)-s| \text { for all } s \in U_{1},
$$

where

$$
\epsilon^{*}=\frac{\epsilon}{1+2 \int_{0}^{1}\left|f^{\prime}(h g(\sigma(t))+(1-h) g(t))\right| \mathrm{d} h} .
$$

Moreover, $f^{\prime}$ is continuous on $\mathbb{R}$ and, therefore, it is uniformly continuous on closed subsets of $\mathbb{R}$. Observing that $g$ is also continuous, because it is $\alpha$-fractional differentiable (see item (i) of Theorem 4 in [5]), there exists a neighbourhood $U_{2}$ of $t$ such that

$$
\left|f^{\prime}(h g(\sigma(t))+(1-h) g(s))-f^{\prime}(h g(\sigma(t))+(1-h) g(t))\right| \leq \frac{\epsilon}{2\left(\epsilon^{*}+\left|T_{\alpha}(g)(t)\right|\right)}
$$

for all $s \in U_{2}$. To see this, note that

$$
\begin{aligned}
|h g(\sigma(t))+(1-h) g(s)-(h g(\sigma(t))+(1-h) g(t))| & =(1-h)|g(s)-g(t)| \\
& \leq|g(s)-g(t)|
\end{aligned}
$$

holds for all $0 \leq h \leq 1$. We then define $U:=U_{1} \cap U_{2}$ and let $s \in U$. For convenience, we put

$$
\gamma=h g(\sigma(t))+(1-h) g(s) \quad \text { and } \quad \beta=h g(\sigma(t))+(1-h) g(t) .
$$

Then we have

$$
\begin{aligned}
\mid[ & (f \circ g)(\sigma(t))-(f \circ g)(s)] t^{1-\alpha}-T_{\alpha}(g)(t)(\sigma(t)-s) \int_{0}^{1} f^{\prime}(\beta) \mathrm{d} h \mid \\
= & \left|t^{1-\alpha}[g(\sigma(t))-g(s)] \int_{0}^{1} f^{\prime}(\gamma) \mathrm{d} h-T_{\alpha}(g)(t)(\sigma(t)-s) \int_{0}^{1} f^{\prime}(\beta) \mathrm{d} h\right| \\
= & \mid\left(t^{1-\alpha}[g(\sigma(t))-g(s)]-(\sigma(t)-s) T_{\alpha}(g)(t)\right) \\
& \times \int_{0}^{1} f^{\prime}(\gamma) \mathrm{d} h+T_{\alpha}(g)(t)(\sigma(t)-s) \int_{0}^{1}\left(f^{\prime}(\gamma)-f^{\prime}(\beta)\right) \mathrm{d} h \mid \\
\leq & \left|t^{1-\alpha}[g(\sigma(t))-g(s)]-(\sigma(t)-s) T_{\alpha}(g)(t)\right| \int_{0}^{1}\left|f^{\prime}(\gamma)\right| \mathrm{d} h \\
& +\left|T_{\alpha}(g)(t)\right||\sigma(t)-s| \int_{0}^{1}\left|f^{\prime}(\gamma)-f^{\prime}(\beta)\right| \mathrm{d} h \\
\leq & \epsilon^{*}|\sigma(t)-s| \int_{0}^{1}\left|f^{\prime}(\gamma)\right| \mathrm{d} h+\left[\epsilon^{*}+\left|T_{\alpha}(g)(t)\right|\right]|\sigma(t)-s| \int_{0}^{1}\left|f^{\prime}(\gamma)-f^{\prime}(\beta)\right| \mathrm{d} h \\
\leq & \frac{\epsilon}{2}|\sigma(t)-s|+\frac{\epsilon}{2}|\sigma(t)-s| \\
= & \epsilon|\sigma(t)-s| .
\end{aligned}
$$

Therefore, $f \circ g$ is $\alpha$-fractional differentiable at $t$ and (1) holds. 
Let us illustrate Theorem 3.1 with an example.

Example 3.2 Let $g: \mathbb{Z} \rightarrow \mathbb{R}$ and $f: \mathbb{R} \rightarrow \mathbb{R}$ be defined by

$$
g(t)=t^{2} \quad \text { and } \quad f(t)=e^{t} .
$$

Then, $T_{\alpha}(g)(t)=(2 t+1) t^{1-\alpha}$ and $f^{\prime}(t)=e^{t}$. Hence, we have by Theorem 3.1 that

$$
\begin{aligned}
T_{\alpha}(f \circ g)(t) & =\left[\int_{0}^{1} f^{\prime}\left(g(t)+h \mu(t) t^{\alpha-1} T_{\alpha}(g)(t)\right) \mathrm{d} h\right] T_{\alpha}(g)(t) \\
& =(2 t+1) t^{1-\alpha} \int_{0}^{1} e^{t^{2}+h(2 t+1)} \mathrm{d} h \\
& =(2 t+1) t^{1-\alpha} e^{t^{2}} \int_{0}^{1} e^{h(2 t+1)} \mathrm{d} h \\
& =(2 t+1) t^{1-\alpha} e^{t^{2}} \frac{1}{2 t+1}\left[e^{2 t+1}-1\right] \\
& =t^{1-\alpha} e^{t^{2}}\left[e^{2 t+1}-1\right] .
\end{aligned}
$$

Theorem 3.3 (Chain Rule II) Let $\mathbb{T}$ be a timescale. Assume $v: \mathbb{T} \rightarrow \mathbb{R}$ is strictly increasing and $\tilde{\mathbb{T}}:=v(\mathbb{T})$ is also a timescale. Let $w: \tilde{\mathbb{T}} \rightarrow \mathbb{R}, \alpha \in(0,1]$, and $\tilde{T}_{\alpha}$ denote the $\alpha$-fractional derivative on $\tilde{\mathbb{T}}$. If for each $t \in \mathbb{T}^{\kappa}, \tilde{T}_{\alpha}(w)(v(t))$ exists and for every $\epsilon>0$, there is a neighbourhood $V$ of $t$ such that

$$
\left|\tilde{\sigma}(v(t))-v(s)-T_{\alpha}(v)(t)(\sigma(t)-s)\right| \leq \epsilon|\sigma(t)-s| \text { for all } s \in V,
$$

where $\tilde{\sigma}$ denotes the forward jump operator on $\tilde{\mathbb{T}}$, then

$$
T_{\alpha}(w \circ v)(t)=\left[\tilde{T}_{\alpha}(w) \circ v\right](t) T_{\alpha}(v)(t) .
$$

Proof Let $0<\epsilon<1$ be given and define $\epsilon^{*}:=\epsilon\left[1+\left|T_{\alpha}(v)(t)\right|+\left|\tilde{T}_{\alpha}(w)(v(t))\right|\right]^{-1}$. Note that $0<\epsilon^{*}<1$. According to the assumptions, there exist neighbourhoods $U_{1}$ of $t$ and $U_{2}$ of $v(t)$ such that

$$
\left|\tilde{\sigma}(v(t))-v(s)-T_{\alpha}(v)(t)(\sigma(t)-s)\right| \leq \epsilon^{*}|\sigma(t)-s|
$$

for all $s \in U_{1}$ and

$$
\left|[w(\tilde{\sigma}(v(t)))-w(r)] t^{1-\alpha}-\tilde{T}_{\alpha}(w)(v(t))(\tilde{\sigma}(v(t))-r)\right| \leq \epsilon^{*}|\tilde{\sigma}(v(t))-r|
$$

for all $r \in U_{2}$. Let $U:=U_{1} \cap v^{-1}\left(U_{2}\right)$. For any $s \in U$, we have that $s \in U_{1}$ and $v(s) \in U_{2}$ with

$$
\begin{aligned}
\left|[w(v(\sigma(t)))-w(v(s))] t^{1-\alpha}-(\sigma(t)-s)\left[\tilde{T}_{\alpha}(w)(v(t))\right] T_{\alpha}(v)(t)\right| \\
=\mid[w(v(\sigma(t)))-w(v(s))] t^{1-\alpha}-[\tilde{\sigma}(v(t))-v(s)] \tilde{T}_{\alpha}(w)(v(t)) \\
\quad+\left[\tilde{\sigma}(v(t))-v(s)-T_{\alpha}(v)(t)(\sigma(t)-s)\right] \tilde{T}_{\alpha}(w)(v(t)) \mid \\
\leq \epsilon^{*}|\tilde{\sigma}(v(t))-v(s)|+\epsilon^{*}|\sigma(t)-s|\left|\tilde{T}_{\alpha}(w)(v(t))\right| \\
\leq \epsilon^{*}\left[\left|\tilde{\sigma}(v(t))-v(s)-(\sigma(t)-s) T_{\alpha}(v)(t)\right|\right. \\
\left.\quad+|\sigma(t)-s|\left|T_{\alpha}(v)(t)\right|+|\sigma(t)-s|\left|\tilde{T}_{\alpha}(w)(v(t))\right|\right] \\
\leq \epsilon^{*}\left[\epsilon^{*}|\sigma(t)-s|+|\sigma(t)-s|\left|T_{\alpha}(v)(t)\right|+|\sigma(t)-s|\left|\tilde{T}_{\alpha}(w)(v(t))\right|\right] \\
=\epsilon^{*}|\sigma(t)-s|\left[\epsilon^{*}+\left|T_{\alpha}(v)(t)\right|+\left|\tilde{T}_{\alpha}(w)(v(t))\right|\right] \\
\leq \epsilon^{*}\left[1+\left|T_{\alpha}(v)(t)\right|+\left|\tilde{T}_{\alpha}(w)(v(t))\right|\right]|\sigma(t)-s| \\
=\epsilon|\sigma(t)-s| .
\end{aligned}
$$

This proves the claim. 
3.2 Fractional integral inequalities on timescales

The $\alpha$-fractional integral on timescales was introduced in [5, Section 3], where some basic properties were proved. Here we show that the $\alpha$-fractional integral satisfies appropriate fractional versions of the fundamental inequalities of Hölder, Cauchy-Schwarz, Minkowski, Jensen and Hermite-Hadamard.

Theorem 3.4 (Hölder's fractional inequality on timescales) Let $\alpha \in(0,1]$ and $a, b \in \mathbb{T}$. If $f, g, h:[a, b] \rightarrow$ $\mathbb{R}$ are rd-continuous, then

$$
\int_{a}^{b}|f(t) g(t)||h(t)| \Delta^{\alpha} t \leq\left[\int_{a}^{b}|f(t)|^{p}|h(t)| \Delta^{\alpha} t\right]^{\frac{1}{p}}\left[\int_{a}^{b}|g(t)|^{q}|h(t)| \Delta^{\alpha} t\right]^{\frac{1}{q}},
$$

where $p>1$ and $\frac{1}{p}+\frac{1}{q}=1$.

Proof For nonnegative real numbers $A$ and $B$, the basic inequality

$$
A^{1 / p} B^{1 / q} \leq \frac{A}{p}+\frac{B}{q}
$$

holds. Now, suppose, without loss of generality, that

$$
\left[\int_{a}^{b}|f(t)|^{p}|h(t)| \Delta^{\alpha} t\right]\left[\int_{a}^{b}|g(t)|^{q}|h(t)| \Delta^{\alpha} t\right] \neq 0 .
$$

Applying Theorem 2.3 and the above inequality to

$$
A(t)=\frac{|f(t)|^{p}|h(t)|}{\int_{a}^{b}|f(\tau)|^{p}|h(\tau)| \Delta^{\alpha} \tau} \quad \text { and } \quad B(t)=\frac{|g(t)|^{q}|h(t)|}{\int_{a}^{b}|g(\tau)|^{p}|h(\tau)| \Delta^{\alpha} \tau},
$$

and integrating the obtained inequality between $a$ and $b$, which is possible since all occurring functions are $r d$-continuous, we find that

$$
\begin{aligned}
\int_{a}^{b}[A(t)]^{1 / p}[B(t)]^{1 / q} \Delta^{\alpha} t \\
\quad=\int_{a}^{b} \frac{|f(t)||h(t)|^{1 / p}}{\left[\int_{a}^{b}|f(\tau)|^{p}|h(\tau)| \Delta^{\alpha} \tau\right]^{1 / p}} \frac{|g(t)||h(t)|^{1 / q}}{\left[\int_{a}^{b}|g(\tau)|^{q}|h(\tau)| \Delta^{\alpha} \tau\right]^{1 / q}} \Delta^{\alpha} t \\
\quad \leq \int_{a}^{b}\left[\frac{A(t)}{p}+\frac{B(t)}{q}\right] \Delta^{\alpha} t \\
\quad=\int_{a}^{b}\left[\frac{1}{p} \frac{|f(t)|^{p}|h(t)|}{\int_{a}^{b}|f(\tau)|^{p}|h(\tau)| \Delta^{\alpha} \tau}+\frac{1}{q} \frac{|g(t)|^{q}|h(t)|}{\int_{a}^{b}|g(\tau)|^{q}|h(\tau)| \Delta^{\alpha} \tau}\right] \Delta^{\alpha} t \\
\quad=\frac{1}{p} \int_{a}^{b}\left[\frac{|f(t)|^{p}|h(t)|}{\int_{a}^{b}|f(\tau)|^{p}|h(\tau)| \Delta^{\alpha} \tau}\right] \Delta^{\alpha} t+\frac{1}{q} \int_{a}^{b}\left[\frac{|g(t)|^{q}|h(t)|}{\int_{a}^{b}|g(\tau)|^{q}|h(\tau)| \Delta^{\alpha} \tau}\right] \Delta^{\alpha} t \\
\quad \leq \frac{1}{p}+\frac{1}{q} \\
=1 .
\end{aligned}
$$

This directly yields the Hölder inequality (2).

As a particular case of Theorem 3.4, we obtain the following inequality.

Theorem 3.5 (Cauchy-Schwarz's fractional inequality on timescales) Let $\alpha \in(0,1]$ and $a, b \in \mathbb{T}$. If $f, g, h$ : $[a, b] \rightarrow \mathbb{R}$ are $r d$-continuous, then

$$
\int_{a}^{b}|f(t) g(t)||h(t)| \Delta^{\alpha} t \leq \sqrt{\left[\int_{a}^{b}|f(t)|^{2}|h(t)| \Delta^{\alpha} t\right]\left[\int_{a}^{b}|g(t)|^{2}|h(t)| \Delta^{\alpha} t\right]} .
$$


Proof Choose $p=q=2$ in Hölder's inequality (2).

Using Hölder's inequality (2), we can also prove the following result.

Corollary 3.6 Let $\alpha \in(0,1]$ and $a, b \in \mathbb{T}$. If $f, g, h:[a, b] \rightarrow \mathbb{R}$ are $r d$-continuous, then

$$
\int_{a}^{b}|f(t) g(t)||h(t)| \Delta^{\alpha} t \geq\left[\int_{a}^{b}|f(t)|^{p}|h(t)| \Delta^{\alpha} t\right]^{\frac{1}{p}}\left[\int_{a}^{b}|g(t)|^{q}|h(t)| \Delta^{\alpha} t\right]^{\frac{1}{q}},
$$

where $\frac{1}{p}+\frac{1}{q}=1$ and $p<0$ or $q<0$.

Proof Without loss of generality, we may assume that $p<0$ and $q>0$. Set $P=-\frac{p}{q}$ and $Q=\frac{1}{q}$. Then, $\frac{1}{P}+\frac{1}{Q}=1$ with $P>1$ and $Q>0$. From (2) we can write that

$$
\begin{aligned}
& \int_{a}^{b}|F(t) G(t)||h(t)| \Delta^{\alpha} t \\
& \quad \leq\left[\int_{a}^{b}|F(t)|^{P}|h(t)| \Delta^{\alpha} t\right]^{\frac{1}{P}}\left[\int_{a}^{b}|G(t)|^{Q}|h(t)| \Delta^{\alpha} t\right]^{\frac{1}{Q}}
\end{aligned}
$$

for any $r d$-continuous functions $F, G:[a, b] \rightarrow \mathbb{R}$. The desired result is obtained by taking $F(t)=[f(t)]^{-q}$ and $G(t)=[f(t)]^{q}[g(t)]^{q}$ in inequality (3).

Next, we use Hölder's inequality (2) to deduce a fractional Minkowski's inequality on timescales.

Theorem 3.7 (Minkowski's fractional inequality on timescales) Let $\alpha \in(0,1], a, b \in \mathbb{T}$ and $p>1$. If $f, g, h:[a, b] \rightarrow \mathbb{R}$ are rd-continuous, then

$$
\begin{aligned}
& {\left[\int_{a}^{b}|(f+g)(t)|^{p}|h(t)| \Delta^{\alpha} t\right]^{1 / p}} \\
& \leq\left[\int_{a}^{b}|f(t)|^{p}|h(t)| \Delta^{\alpha} t\right]^{\frac{1}{p}}+\left[\int_{a}^{b}|g(t)|^{p}|h(t)| \Delta^{\alpha} t\right]^{\frac{1}{p}} .
\end{aligned}
$$

Proof We apply Hölder's inequality (2) with $q=p /(p-1)$ and items (i) and (v) of Theorem 2.3 to obtain

$$
\begin{aligned}
\int_{a}^{b} \mid(f & +g)\left.(t)\right|^{p}|h(t)| \Delta^{\alpha} t \\
= & \int_{a}^{b}|(f+g)(t)|^{p-1}|(f+g)(t)||h(t)| \Delta^{\alpha} t \\
\leq & \int_{a}^{b}|f(t)||(f+g)(t)|^{p-1}|h(t)| \Delta^{\alpha} t+\int_{a}^{b}|g(t)||(f+g)(t)|^{p-1}|h(t)| \Delta^{\alpha} t \\
\leq & {\left[\int_{a}^{b}|f(t)|^{p}|h(t)| \Delta^{\alpha} t\right]^{\frac{1}{p}}\left[\int_{a}^{b}|(f+g)(t)|^{(p-1) q}|h(t)| \Delta^{\alpha} t\right]^{\frac{1}{q}} } \\
& +\left[\int_{a}^{b}|g(t)|^{p}|h(t)| \Delta^{\alpha} t\right]^{\frac{1}{p}}\left[\int_{a}^{b}|(f+g)(t)|^{(p-1) q}|h(t)| \Delta^{\alpha} t\right]^{\frac{1}{q}} \\
= & {\left[\int_{a}^{b}|(f+g)(t)|^{p}|h(t)| \Delta^{\alpha} t\right]^{\frac{1}{q}} } \\
& \times\left[\left[\int_{a}^{b}|f(t)|^{p}|h(t)| \Delta^{\alpha} t\right]^{\frac{1}{p}}+\left[\int_{a}^{b}|g(t)|^{p}|h(t)| \Delta^{\alpha} t\right]^{\frac{1}{p}}\right)
\end{aligned}
$$

Dividing both sides of the obtained inequality by $\left[\int_{a}^{b}|(f+g)(t)|^{p}|h(t)| \Delta^{\alpha} t\right]^{\frac{1}{q}}$, we arrive at the Minkowski inequality (4). 
Jensen's classical inequality relates the value of a convex/concave function of an integral to the integral of the convex/concave function. We prove a generalization of such relation for the BHT fractional calculus on timescales.

Theorem 3.8 (Generalized Jensen's fractional inequality on timescales) Let $\mathbb{T}$ be a timescale, $a, b \in \mathbb{T}$ with $a<b, c, d \in \mathbb{R}, \alpha \in(0,1], g \in C([a, b] \cap \mathbb{T} ;(c, d))$ and $h \in C([a, b] \cap \mathbb{T} ; \mathbb{R})$ with

$$
\int_{a}^{b}|h(s)| \Delta^{\alpha} s>0 .
$$

- If $f \in C((c, d) ; \mathbb{R})$ is convex, then

$$
f\left(\frac{\int_{a}^{b} g(s)|h(s)| \Delta^{\alpha} s}{\int_{a}^{b}|h(s)| \Delta^{\alpha} s}\right) \leq \frac{\int_{a}^{b} f(g(s))|h(s)| \Delta^{\alpha} s}{\int_{a}^{b}|h(s)| \Delta^{\alpha} s} .
$$

- If $f \in C((c, d) ; \mathbb{R})$ is concave, then

$$
f\left(\frac{\int_{a}^{b} g(s)|h(s)| \Delta^{\alpha} s}{\int_{a}^{b}|h(s)| \Delta^{\alpha} s}\right) \geq \frac{\int_{a}^{b} f(g(s))|h(s)| \Delta^{\alpha} s}{\int_{a}^{b}|h(s)| \Delta^{\alpha} s} .
$$

Proof We start by proving (5). Since $f$ is convex, for any $t \in(c, d)$ there exists $a_{t} \in \mathbb{R}$ such that

$$
a_{t}(x-t) \leq f(x)-f(t) \text { for all } x \in(c, d) .
$$

Let

$$
t=\frac{\int_{a}^{b} g(s)|h(s)| \Delta^{\alpha} s}{\int_{a}^{b}|h(s)| \Delta^{\alpha} s} .
$$

It follows from (7) and item (v) of Theorem 2.3 that

$$
\begin{aligned}
& \int_{a}^{b} f(g(s))|h(s)| \Delta^{\alpha} s-\left(\int_{a}^{b}|h(s)| \Delta^{\alpha} s\right) f\left(\frac{\int_{a}^{b} g(s)|h(s)| \Delta^{\alpha} s}{\int_{a}^{b}|h(s)| \Delta^{\alpha} s}\right) \\
& =\int_{a}^{b} f(g(s))|h(s)| \Delta^{\alpha} s-\left(\int_{a}^{b}|h(s)| \Delta^{\alpha} s\right) f(t) \\
& =\int_{a}^{b}(f(g(s))-f(t))|h(s)| \Delta^{\alpha} s \\
& \geq a_{t} \int_{a}^{b}(g(s)-t)|h(s)| \Delta^{\alpha} s \\
& =a_{t}\left(\int_{a}^{b} g(s)|h(s)| \Delta^{\alpha} s-t \int_{a}^{b}|h(s)| \Delta^{\alpha} s\right) \\
& =a_{t}\left(\int_{a}^{b} g(s)|h(s)| \Delta^{\alpha} s-\int_{a}^{b} g(s)|h(s)| \Delta^{\alpha} s\right) \\
& =0 .
\end{aligned}
$$

This proves (5). To prove (6), we simply observe that $F(x)=-f(x)$ is convex (because we are now assuming $f$ to be concave) and then we apply inequality (5) to function $F$.

We end with an application of Theorem 3.8. 
Theorem 3.9 (A weighted fractional Hermite-Hadamard inequality on timescales) Let $\mathbb{T}$ be a timescale, $a, b \in \mathbb{T}$ and $\alpha \in(0,1]$. Let $f:[a, b] \rightarrow \mathbb{R}$ be a continuous convex function and let $w: \mathbb{T} \rightarrow \mathbb{R}$ be $a$ continuous function such that $w(t) \geq 0$ for all $t \in \mathbb{T}$ and $\int_{a}^{b} w(t) \Delta^{\alpha} t>0$. Then,

$$
f\left(x_{w, \alpha}\right) \leq \frac{1}{\int_{a}^{b} w(t) \Delta^{\alpha} t} \int_{a}^{b} f(t) w(t) \Delta^{\alpha} t \leq \frac{b-x_{w, \alpha}}{b-a} f(a)+\frac{x_{w, \alpha}-a}{b-a} f(b),
$$

where $x_{w, \alpha}=\frac{\int_{a}^{b} t w(t) \Delta^{\alpha} t}{\int_{a}^{b} w(t) \Delta^{\alpha} t}$.

Proof For every convex function one has

$$
f(t) \leq f(a)+\frac{f(b)-f(a)}{b-a}(t-a) .
$$

Multiplying this inequality with $w(t)$, which is nonnegative, we get

$$
w(t) f(t) \leq f(a) w(t)+\frac{f(b)-f(a)}{b-a}(t-a) w(t) .
$$

Taking the $\alpha$-fractional integral on both sides, we can write that

$$
\int_{a}^{b} w(t) f(t) \Delta^{\alpha} t \leq \int_{a}^{b} f(a) w(t) \Delta^{\alpha} t+\int_{a}^{b} \frac{f(b)-f(a)}{b-a}(t-a) w(t) \Delta^{\alpha} t,
$$

which implies

$$
\begin{aligned}
& \int_{a}^{b} w(t) f(t) \Delta^{\alpha} t \\
& \quad \leq f(a) \int_{a}^{b} w(t) \Delta^{\alpha} t+\frac{f(b)-f(a)}{b-a}\left(\int_{a}^{b} t w(t) \Delta^{\alpha} t-a \int_{a}^{b} w(t) \Delta^{\alpha} t\right)
\end{aligned}
$$

that is,

$$
\frac{1}{\int_{a}^{b} w(t) \Delta^{\alpha} t} \int_{a}^{b} f(t) w(t) \Delta^{\alpha} t \leq \frac{b-x_{w, \alpha}}{b-a} f(a)+\frac{x_{w, \alpha}-a}{b-a} f(b) .
$$

We have just proved the second inequality of (8). For the first inequality of (8), we use (5) of Theorem 3.8 by taking $g: \mathbb{T} \rightarrow \mathbb{T}$ defined by $g(s)=s$ for all $s \in \mathbb{T}$ and $h: \mathbb{T} \rightarrow \mathbb{R}$ given by $h=w$.

Note that if in Theorem 3.9 we consider a concave function $f$ instead of a convex one, then the inequalities of (8) are reversed.

Acknowledgements Torres was partially supported by the Portuguese Foundation for Science and Technology (FCT), through the Center for Research and Development in Mathematics and Applications (CIDMA), within project UID/MAT/04106/2013. The authors are greatly indebted to two referees for their several useful suggestions and valuable comments.

Open Access This article is distributed under the terms of the Creative Commons Attribution 4.0 International License (http:// creativecommons.org/licenses/by/4.0/), which permits unrestricted use, distribution, and reproduction in any medium, provided you give appropriate credit to the original author(s) and the source, provide a link to the Creative Commons license, and indicate if changes were made.

\section{References}

1. Bastos, N.R.O.; Ferreira, R.A.C.; Torres, D.F.M.: Necessary optimality conditions for fractional difference problems of the calculus of variations. Discrete Contin. Dyn. Syst. 29(2), 417-437 (2011)

2. Bastos, N.R.O.; Mozyrska, D.; Torres, D.F.M.: Fractional derivatives and integrals on time scales via the inverse generalized Laplace transform. Int. J. Math. Comput. 11(J11), 1-9 (2011)

3. Benkhettou, N.; Brito da Cruz, A.M.C.; Torres, D.F.M.: A fractional calculus on arbitrary time scales: fractional differentiation and fractional integration. Signal Process. 107, 230-237 (2015)

4. Benkhettou, N.; Brito da Cruz, A.M.C.; Torres, D.F.M.: Nonsymmetric and symmetric fractional calculi on arbitrary nonempty closed sets. Math. Methods Appl. Sci. 39(2), 261-279 (2016)

5. Benkhettou, N.; Hassani, S.; Torres, D.F.M.: A conformable fractional calculus on arbitrary time scales. J. King Saud Univ. Sci. 28(1), 93-98 (2016)

6. Bohner, M.; Peterson, A.: Dynamic Equations on Time Scales. Birkhäuser Boston, Boston (2001) 